\title{
Zwischen Selbstverwirklichung und Selbsttranszendenz - Menschliche Selbstentwürfe und die ethische Frage nach dem Guten
}

DAGMAR FENNER

\section{Der Mensch - ein Besonderes Tier}

Was ist der Mensch? Man kann den Menschen im Zeichen der physiologischen Anthropologie von außen beschreiben und als Objekt betrachten. Es lässt sich dann etwa feststellen, dass der Mensch ausgeprägte Artikulationsorgane, freigelegte Hände, eine spezifisch ausgebildete Großhirnrinde und ein außerordentlich großes Hirnvolumen besitzt. Mit diesen biologischen Merkmalen beschreibt man den Menschen als Naturwesen. Die Naturwissenschaft kann den Menschen aber nie vollständig beschreiben und erfassen. Denn der Mensch ist ein sehr besonderes Tier und viel mehr als bloßes Naturwesen. Aufgrund der Ausstattung mit bewusstem Denken und Reflexionsvermögen hat sich das Tier Mensch zu einem Kulturwesen mit einem ungeahnten Spielraum an Handlungs- und Lebensmöglichkeiten entwickelt. Aufgrund seiner hochentwickelten Sprachfähigkeit und der hirnphysiologischen Besonderheiten lebt der Mensch nicht wie das Tier bloß in einer unmit- 
telbaren Verzahnung mit der Umwelt. Er erfasst die Welt nicht ausschließlich in Bezug auf die eigenen vitalen Interessen in Kategorien wie »Fressbares « oder »Nicht-Fressbares«, »Geschlechtspartner« oder »Geschlechtsgenosse«. Vielmehr nimmt er die Umwelt sachlicher und »weltoffen« (vgl. Scheler 1991: 42) wahr und kann sein Leben voraussehend und zwecksetzend frei gestalten. »Der Mensch lebt nicht, sondern er führt sein Leben«, so hat es der Anthropologe Arnold Gehlen (1986: 165) auf den Punkt gebracht.

In den Geisteswissenschaften interessiert der Mensch nicht als ein Objekt wie in den Naturwissenschaften. Vielmehr tritt er als Subjekt in den Blickpunkt: als ein Kulturwesen, das die empirischen Gegebenheiten deuten und sich auf dieser Grundlage ein >Bild des Menschen< machen muss. Aus Sicht einer pragmatischen Anthropologie oder Ethik interessiert, wozu die beschriebenen Charaktermerkmale den Menschen befähigen und was er damit machen soll. Es ist so gesehen gerade das Typische für den Menschen, dass er sich erst selbst definieren und entwerfen muss. Der Mensch ist das »noch nicht festgestellte Thier«, heißt es bei Friedrich Nietzsche (1980: 81). »Der Mensch ist zuerst ein Entwurf«, lesen wir bei Jean-Paul Sartre: »Der Mensch ist nichts anderes, als wozu er sich macht« (Sartre 1989: 11). Und sein wohl bekanntestes Diktum: »Der Mensch ist verurteilt, frei zu sein« (ebd.: 16). Angesichts der riesigen Spannbreite menschlicher Möglichkeiten wird der Mensch sich selbst zum Rätsel und Problem: Was soll er aus sich machen? Wie kann er das Gute realisieren und das Böse meiden? Welches Handeln ist ethisch richtig?

\section{Zwei Perspektiven in der Ethik}

Allein aufgrund unserer Reflexionsfähigkeit stellen wir uns ständig die Frage, wie wir handeln sollen. Denn meistens steht uns eine Vielzahl von Handlungsmöglichkeiten zur Verfügung, zwischen denen wir auswählen müssen. Man denke etwa an folgende Entscheidungssituation: Sie haben sich vielleicht lange überlegt, ob Sie an diesem Abend zu 
diesem Vortrag über Selbstverwirklichung kommen sollen oder ob Sie einen Artikel zu Ende schreiben oder mit Ihrer Familie zu Abend essen sollen. Es geht dann um die Grundfrage der praktischen Philosophie: Wie soll ich handeln? Viele praktische Grundfragen in alltäglichen Entscheidungssituationen lassen sich dabei vertiefen, bis die individuelle Lebenssituation als Ganze in den Blick rückt. So könnten Sie sich in unserem Beispiel etwa gefragt haben, wie wichtig in Ihrem Leben eigentlich die philosophisch-schöngeistige Weiterbildung ist. Oder Sie überlegten sich grundsätzlich, ob Sie bei Ihrem zeitintensiven Job und dem ausgefüllten Freizeitprogramm nicht Ihre Familie allzu stark vernachlässigen. Sobald wir solche weiterführenden praktischen Überlegungen über die Gestaltung unserer Freizeit oder die Gewichtung von Beruf und Familie anstellen, stoßen wir zu einer zweiten, umfassenderen praktischen Grundfrage vor: Wie soll ich leben? Was ist mir im Leben wichtig? Wer will ich eigentlich sein?

Lassen Sie mich ganz kurz für alle Nichtphilosophen erläutern: Während sich die theoretische Philosophie mit dem befasst, was ist, interessiert sich die praktische Philosophie oder Ethik dafür, was getan werden soll. Während die theoretische Philosophie in Bezug auf das Seiende nach Wahrheit strebt, entwirft die Ethik das Ideal des Guten für das menschliche Handeln. Ethik ist die Disziplin der praktischen Philosophie, die anhand allgemeiner Prinzipien oder Beurteilungskriterien zu begründen versucht, welches Handeln gut oder richtig ist. Die ethische Frage, wie Menschen handeln und wie sie sich selbst und ihr Leben entwerfen sollen, kann dabei grundsätzlich auf zwei unterschiedliche Weisen verstanden werden. Sie stellt sich zum einen mit Blick auf die persönliche Lebensführung und die Eigeninteressen des jeweiligen Individuums. Es geht dann darum, wie der Einzelne die persönlichen Wünsche und Ziele am besten erreicht. In Frage steht das für das Individuum Gute, sein gutes Leben, letztlich sein persönliches Glück. Die philosophischen Reflexionen von dieser Warte aus bezeichnet man oft als Individualethik oder Strebensethik.

Ein anderes Bild zeigt sich aus der Perspektive der Sozialethik, Sollensethik oder Moralphilosophie: Hier steht nicht das für ein Individu- 
um Gute im Zentrum, sondern das für eine bestimmte Gemeinschaft Gute. Gut bzw. richtig ist ein Handeln dann, wenn der Handelnde nicht nur die eigenen, sondern auch die Interessen aller vom Handeln Betroffenen angemessen berücksichtigt. Anstelle des Selbstverhältnisses des Einzelnen zu sich selbst gerät hier das Zusammenleben der Menschen in den Blick; anstelle des Eigen-Wollens das moralische Sollen. Das Ideal der Sollensethik oder Moralphilosophie ist nicht das Glück, sondern die Gerechtigkeit.

Sie haben es natürlich längst bemerkt: Auf diese Weise sind im Grunde die beiden Pole umschrieben, die im Titel meines Vortrags stehen: Selbstverwirklichung und Selbsttranszendenz. Selbsttranszendenz meint die genuin menschliche Fähigkeit, die Ich-Perspektive zu überwinden oder eben zu transzendieren, von lateinisch transcendere: hinüberschreiten oder übersteigen. In einem spirituellen, religiösen Sinn meint Selbsttranszendenz das Überschreiten der Grenzen des eigenen Geistes oder Egos und das Eintreten in höhere Formen der Realität. Doch davon soll heute nicht die Rede sein, da für diese Dimension eher Theologen oder Religionswissenschaftler zuständig wären. Ich werde mich daher auf die ethische Dimension beschränken: Innerhalb der Ethik geht es in der Sozialethik oder Moralphilosophie um diese Selbsttranszendenz. Denn der objektive oder unparteiische Standpunkt der Moral verlangt, den eigenen Interessenstandpunkt zu transzendieren und stellvertretend die Standpunkte aller anderen Menschen, aber auch der Tiere oder der Natur einzunehmen. Man soll dann nicht das tun, was nur im eigenen Interesse ist, sondern alle Bedürfnisse und Interessen gleichermaßen berücksichtigen. Um Selbstverwirklichung geht es hingegen in der Individualethik oder der Ethik des guten Lebens. Denn es herrscht heute in der Philosophie und in der breiten Öffentlichkeit ein weitgehender Konsens darüber, dass der Weg zum Glück als Letztziel eines guten Lebens nur auf dem Weg über die Selbstverwirklichung zu erreichen sei.

Die Menschen können sich bei ihren Selbstentwürfen also grundsätzlich entweder an dem für sie persönlich Guten oder an dem für ihre Um- und Mitwelt Guten oder dem Allgemeinwohl orientieren. In 
einem ersten Teil meines Vortrags werde ich Ihnen zwei verschiedene Konzepte von Selbstverwirklichung vorstellen und kritisch erläutern. Danach soll in einem zweiten Teil gefragt werden, wie sich die beiden Pole der Selbstverwirklichung und Selbsttranszendenz zueinander verhalten. Ist aus ethischer Sicht dem moralischen Standpunkt eindeutig der Vorrang zu geben? Ist das individualethische Streben nach individueller Selbstverwirklichung nicht egoistisch und unmoralisch?

\section{Zwei Modelle der SelbstVerwirklichung}

Die menschliche Freiheit, sich selbst entwerfen zu können, wurde geschichtlich gesehen erst eigentlich in der Renaissance entdeckt. Anders als in Antike und Mittelalter versuchte man den Menschen in der Renaissance nicht länger von der Gesamtordnung des Kosmos oder der göttlichen Schöpfung her zu deuten, sondern aus sich selbst heraus zu verstehen. Einen massiven Aufschwung erlebten Programme zur Selbstfindung, Selbstentfaltung und Selbstverwirklichung dann erst in den späten 1960er Jahren.

Diese Konjunktur verdankt sich zum einen verschiedenen Individualisierungsschüben im Laufe der Neuzeit und auch dem Entstehen einer liberalen, kapitalistischen Marktwirtschaft. Zum anderen hat ein erheblicher Religions- und Traditionsverlust dazu geführt, dass in unserer Gesellschaft nicht mehr eine einzige vorgegebene Lebensform als die richtige gilt. Für Christen beispielsweise war es Pflicht, ein gottesfürchtiges, bibeltreues Leben zu führen, sich in der Kirchgemeinde zu engagieren und sich karitativ zu betätigen. Noch für die Generation meiner Großeltern war es selbstverständlich, dass Frauen keine Ausbildung bekommen, sondern heiraten und Kinder großziehen. Die Männer hingegen erlernten den Beruf ihres Vaters und übernahmen gegebenenfalls den väterlichen Hof oder Betrieb. Man hatte nicht die Qual der Wahl, die wir heute angesichts einer unendlichen Vielfalt an Lebensmöglichkeiten haben. Nicht zuletzt spricht man in der Psychologie von einer neuen, überaus permissiven, also >zulassenden<, ent- 
gegenkommenden Erziehung. Bei diesem Erziehungsstil der >neuen Verwöhnung richten sich die Erziehungspersonen primär nach den Wünschen und Bedürfnissen der Kinder (vgl. Kleiter 1999: 467).

Je weniger die Menschen vorgegebenen Pflichten und Aufgaben nachkommen müssen, desto mehr scheinen sie nur noch sich selbst verwirklichen zu müssen. Je lauter aber der allgemeinmenschliche Ruf nach Selbstverwirklichung erschallt und je breiter sich das entsprechende Angebot an Lebenshilfeliteratur und Kursen gestaltet, desto undifferenzierter werden die Vorstellungen darüber, was denn hier als $>$ Selbst< eigentlich gefunden oder realisiert werden soll. Es gilt also zu klären: Was heißt eigentlich Selbstverwirklichung oder Selbstaktualisierung?

Grundsätzlich kann man zwei Modelle der Selbstverwirklichung unterscheiden (vgl. Gewirth 1998: 13f.): Beim ersten Modell begreift man Selbstverwirklichung als Entfaltung von bestimmten menschlichen Kräften oder Fähigkeiten. Wir nennen es den Fähigkeiten-Ansatz. Die Vertreter dieses Modells gehen meist von objektiven Kriterien oder Werten aus: von dem, was für einen Menschen objektiv gesehen gut ist im Sinne des allgemeinen Menschseins. Ziel ist dann eine überindividuelle Wesensrealisierung. Im zweiten Modell meint Selbstverwirklichung hingegen die Erfüllung der tiefsten Wünsche oder Bestrebungen eines Handlungssubjekts. Es lässt sich daher von einem wunschtheoretischen Ansatz sprechen. Hier scheint Selbsterfüllung stärker eine subjektive Angelegenheit zu sein. Motto ist hier nicht >Werde, der Du bist<, sondern eher: >Erfinde Dich selbst!< (Vgl. Horn 2014: 81)

Beginnen wir mit dem ersten Modell, dem Fähigkeiten-Ansatz der Selbstverwirklichung. Es geht auf Platon und Aristoteles zurück und wurde im 20. Jahrhundert von den humanistischen Psychologen reaktiviert. Wichtige Vertreter sind Erich Fromm, Kurt Goldstein und Abraham Maslow. Mit Unterstützung der humanistischen Selbst-Theoretiker wurde in den 1970er Jahren eine regelrechte $>$ Me-Decade<, ein >Zeitalter des Ichく ausgerufen. Eine ganze Generation von Selbstverwirklichern strömte in Selbsterfahrungsseminare und Therapieräume. 
Selbstverwirklichung ist aus dieser Sicht der Prozess der optimalen Entfaltung der menschlichen Potenzialitäten, d.h. seiner faktischen Bedürfnisse, Fähigkeiten und Begabungen. Das zu verwirklichende >Selbst< wäre die Gesamtheit von angeborenen, natürlichen Kräften und Fähigkeiten, die auf Entwicklung hin angelegt sind. In den meisten Fällen scheint es um eine wertneutrale Aktualisierung all dieser Fähigkeiten zu gehen. Manchmal spricht man auch von den >besten Potenzen<, ohne dass klar wäre, welche das sein sollen (vgl. Gerhardt 1989: 38). Gern bemüht wird dabei die Samenkorn-Analogie: Die Entwicklung eines Kindes erfolge wie das Wachstum eines Samenkorns.

Diese Samenkorn-Analogie ist aus meiner Sicht jedoch äußerst irreführend. Denn die optimale Entwicklung des Kindes ist natürlich etwas ganz anderes als das Wachstum des Samenkorns. Sie ist anders als jene auf sorgfältige Erziehung und kulturelle Sozialisation angewiesen. Im Gegensatz zum biologischen Programm von Pflanzen oder den tierischen Instinkten sind die natürlichen Kräfte oder Tendenzen im Menschen äußerst vage und unbestimmt. Darüber hinaus können sich die individuellen Bedürfnisse und Talente im Widerstreit miteinander befinden. Gewisse lassen sich vielleicht nur zeitlich nacheinander oder in einem Menschenleben gar nicht erfüllen. Auch können die wertmäßig zunächst indifferenten Anlagen der Menschen ebenso zu schlechten wie zu guten Zwecken eingesetzt werden. Studien zu eineiigen Zwillingen ergaben, dass sich aus dem gleichen genetischen Material völlig unterschiedliche Persönlichkeiten und Lebensmöglichkeiten entwickeln können. So wurde beispielsweise von Zwillingsbrüdern mit der angeborenen Charaktereigenschaft >Raffinement< der eine ein raffinierter Krimineller, der andere ein ebenso raffinierter Kriminologe (vgl. Frankl 1994: 90f.). Die angeborenen Charakteranlagen sind sozusagen $>$ wertneutral<, weder Tugenden noch Laster.

Das simple biologische Schema, demzufolge aus einem Apfelkern ein Apfelbaum oder aus einem menschlichen Embryo ein erwachsener Mensch wird, zeichnet also ein schiefes Bild vom menschlichen Entwicklungsprozess. Zum einen ist eine klare Bewertung zwischen verschiedenen Fähigkeiten und Anlagen unausweichlich. Zum anderen 
müssen die positiv bewerteten natürlichen Tendenzen sorgfältig gefördert und kultiviert werden. Wenn der Mensch nur das zu werden brauchte, was er immer schon potenziell ist, könnte man die für den Menschen typische geistige Dimension wegkürzen. Sobald wir den Selbstverwirklichungsprozess bewertend und regulierend kontrollieren, handelt es sich aber nicht mehr um ein potenziell schon vorhandenes Selbst, das auf ganz >natürliche< Weise entfaltet wird (vgl. Gewirth 1998: 12).

Werfen wir einen kurzen Blick auf Aristoteles, der als Urvater des Fähigkeiten-Modells betrachtet werden kann: Obwohl auch Aristoteles von angeborenen menschlichen Potenzen oder Fähigkeiten ausgeht, setzt er sich ausführlich mit den Vor- und Nachteilen des Auslebens bestimmter Anlagen auseinander (vgl. ebd.: 13). In der Antike forderte man die Ausbildung von Tugenden oder festen Charakterhaltungen, um menschliche Anlagen gezielt zu fördern. Diese festen inneren Haltungen sollen es dem Menschen ermöglichen, in jeder Situation das ethisch Richtige zu tun. Zwischen Potenzialität und Aktualität schiebt sich also der Erwerb von Tugenden.

Seit den Anfängen der philosophischen Überlegungen zum Selbstverwirklichungsmodell hat man sich primär dafür interessiert, welches denn die >wahren< oder >eigentlichen< Fähigkeiten des Menschen sind (vgl. Höffe 2004: 41f.). In der Antike führte man diese Frage zurück auf die Frage nach der >eigentümlichen Leistung $<$, griechisch `Ergon< des Menschen. Nicht nur alle Gegenstandsklassen und alle Berufsgattungen wie etwa Messer und Tische oder Bildhauerei und Tischlerei hätten ihre artspezifische Funktionsfähigkeit oder Hervorbringung. Auch dem Menschen als solchem müsse eine typische und damit höherrangige Tätigkeit zukommen. Aristoteles erblickte wie sein Lehrer Platon das spezifische menschliche >Ergon< in der Tätigkeit der Vernunft, im reinen Denken (vgl. Aristoteles 1991: 1097b, 21-1098a, 20). Die wahre menschliche Selbstverwirklichung besteht daher nach Aristoteles in der Beschäftigung mit kontemplativen theoretischen Wissenschaften wie Philosophie, Mathematik oder theoretischer Physik. 
Nun blieb auch Aristoteles nicht verborgen, dass zum einen nicht alle Menschen die Fähigkeit und Disziplin haben, sich ganz auf die Förderung ihrer intellektuellen Kompetenzen zu konzentrieren. Zum zweiten sind auch diese theoretisch begabten Köpfe keine reinen Intelligenzwesen. Vielmehr haben auch sie noch andere Bedürfnisse und Interessen und pflegen zu diesem Zweck den Umgang mit anderen Menschen. Sie sollen daher auch für das Zusammenleben wichtige menschliche Fähigkeiten in der kooperativen Praxis einüben. Sie benötigen sozialethisch relevante charakterliche Tugenden wie Großzügigkeit, Wohlwollen und Gerechtigkeit. Die intellektuellen Tugenden sollen also durch die ethischen Tugenden, die theoretische Lebensform durch die moralisch-politische Lebensform ergänzt werden.

Die aristotelische Anleitung zur bestmöglichen Verwirklichung der spezifisch menschlichen Fähigkeiten hatte eine große, lang anhaltende Wirkung. Allerdings steht außer Zweifel, dass es bedeutend mehr Möglichkeiten menschlicher Selbstverwirklichung gibt als die von Aristoteles vorgeschlagene theoretische und ethisch-politische (vgl. Höffe 2004: 42): Für religiöse Kulturen ist es die demutsvolle Hinwendung zu Gott, für kriegerische das tapfere Heldentum. In der Neuzeit wuchs die Skepsis gegenüber einem Konzept, das von vornherein festlegt, welche Fähigkeiten ein jeder Mensch objektiv betrachtet zur Entfaltung bringen soll. Als bleibende Erkenntnis aus der Auseinandersetzung mit Aristoteles könnte man festhalten: Die menschliche Vernunft ist das entscheidende Vermögen bei der Auswahl, Ordnung und Ausbildung aller menschlichen Fähigkeiten und Lebensmöglichkeiten. Zudem muss sich ein Modell menschlicher Selbstverwirklichung mit der sozialen Dimension einer ethisch-politischen Lebensform auseinandersetzen. Heute verbindet man mit Selbstverwirklichung jedoch kaum mehr die Verwirklichung wesensmäßiger, natürlicher Fähigkeiten oder artspezifische Leistungen im Sinne des antiken Ergon. Der Trend in der Gegenwart geht eindeutig hin zum zweiten Modell der Selbstverwirklichung, dem wunschtheoretischen Ansatz: Selbstverwirklichung wird verstanden als Erfüllung tiefer Wünsche, Lebensziele oder identitätsstiftender Ideale. 
Ich komme damit also zum zweiten Modell menschlicher Selbstverwirklichung: Das zu verwirklichende >Selbst $<$ ist hier nichts natürlich Vorgegebenes, sondern ein idealer Entwurf seiner selbst. In der Philosophie nennt man dieses >Selbst< ein >normatives Selbst< oder >normatives Selbstbild $\measuredangle$. »Normativ ist dasjenige Selbst, von dem wir uns wählend verstehen, der Entwurf, auf den hin wir uns verwirklichen wollen, das eigentliche, wahre, selbsthafte Selbst, das Selbst im Selbst; faktisch das, als welches wir uns jeweils vorfinden.« (Krämer 1978: 111f.) Es heißt >normatives $<$, also >wertendes< Selbst, weil man in einem reflexiven Vorgang der Interpretation und Auswahl bestimmte Fähigkeiten oder Neigungen als wertvoll bestimmt. Der normative Selbstentwurf ist prospektiv, weil er sich auf die Zukunft bezieht und festlegt, in welche Richtung die Entwicklung gehen soll. Es handelt sich bei dem in die Zukunft projizierten $>$ Selbst< allerdings nicht um eine >creatio ex nihilo<, eine Schöpfung aus dem Nichts. Man bezieht sich sehr wohl auf ein >faktisches Selbst< oder >empirisches Selbst<, das die genetischen Anlagen, die angeborene psychische Charakterdisposition, aber auch den eigenen Körper, die religiös-kulturelle Einbettung, familiäre Herkunft und die sozialen Rollen umfasst. Es ist individualethisch gesehen nicht ratsam, ganz unabhängig von empirischen Gegebenheiten etwas Unmögliches oder Phantastisches werden zu wollen, z.B. Nero oder Cäsar. Denn dann ist die Gefahr groß, dass das >normative Selbst< ein bloßes Phantasieprodukt bleibt und nicht in die Realität umgesetzt werden kann.

Das >empirische Selbst< ist sozusagen das passive Moment im $>$ menschlichen Selbst $<$, das in der Sozialphilosophie >me $<$ genannt wird. Das aktive Moment, auch das $>\mathrm{I}<$ (Mead) oder >reine Selbst< (James) genannt, steht für die menschliche Möglichkeit, bewusst und reflexiv Stellung zu nehmen zu allen charakterlichen, biographischen und situativen Gegebenheiten. Aufgrund dieser beiden Momente des aktiven geistigen $>\mathbb{K}<$ und des passiven empirischen $>$ me $<$ ist der Mensch nach Sartre dazu verurteilt, immer das zu sein, was er nicht ist, und nie das zu sein, was er ist (vgl. Sartre 1991: 267). Also nochmals: Er ist nicht, was er ist - und ist, was er nicht ist. Das klingt paradox: Der Mensch 
ist eben immer mehr als das, was von ihm bereits in Erscheinung getreten ist - also beispielsweise seine Vergangenheit, sein Körper oder sein gewordener Charakter. Der Mensch kann dieses pure Dasein dank seiner Reflexionsfähigkeit überschreiten, sich davon distanzieren oder ihm eine bestimmte Bedeutung innerhalb seines Selbstentwurfs geben. In diesem Sinn ist er, was er nicht ist.

Aus einem ausgearbeiteten oder ausformulierten normativen Selbstentwurf ergibt sich der Lebensplan oder die Lebensform eines Menschen. Dabei liegt der Zeitpunkt dieser ursprünglichen Selbstwahl und des persönlichen Lebenskonzepts am Ende der pubertären Krise. Während der Lebensplan mannigfaltige Ziele in eine zeitliche und hierarchische Ordnung bringt, stehen im Zentrum von Selbstentwürfen eher Ideale. Ideale sind nicht wie die Lebensziele oder Projekte auf die Gestaltung der äußeren Welt ausgerichtet, sondern betreffen mehr die Seinsweise des Subjekts. Ideale wie >Schönheit< oder > Weisheit< sind weniger konkret, weniger realitätsorientiert als berufliche Ziele wie eine Karriere als Top-Model oder Philosophieprofessorin. Da der Selbstentwurf genauso wie der Lebensplan in die Tiefen des Unbewussten hinabgeübt wird, muss er dabei auch gar nicht immer bewusst sein, um wirksam zu sein. Das aktive >reine Selbst< kann aber jederzeit reflexive Distanz nehmen zu diesem Entwurf. Ihm kommt die permanente Aufgabe der Selbstkontrolle und Selbststeuerung zu. Im Krisenfall muss es eine Korrektur oder eine Neuinterpretation der Lebensund Selbstentwürfe vornehmen. Entweder wenn sie sich mit Blick auf die eigenen biologischen, psychischen oder charakterlichen Fähigkeiten als inadäquat entpuppen oder wenn sich die Umweltgegebenheiten unerwartet verändern. Es empfiehlt sich daher, sich die Selbstreflexion zur Gewohnheit zu machen. 


\section{Die Wunsch- oder Zieltheorie}

Sie fragen sich jetzt vielleicht: Wie sollen wir denn unsere Ideale und Lebensziele auswählen, um ein gutes und glückliches Leben zu führen? In der Philosophie ist es die Wunsch- oder Zieltheorie des guten Lebens, die sich mit solchen Fragen beschäftigt. Gemäß diesen Theorien bemisst sich ein gutes Leben daran, dass in ihm die meisten, die wesentlichen oder möglichst viele Wünsche oder Ziele des Subjekts realisiert werden können. Die Grundmaxime dieser Position lautet entsprechend: >Lebe das Leben, bei dem möglichst viele Deiner Wünsche oder Ziele in Erfüllung gehen! < Es ist empirisch kaum zu leugnen, dass jeder Mensch Wünsche hat, zu denen er wertend Stellung beziehen kann. Erfahrungsgemäß möchte jeder Mensch, dass möglichst viele seiner Wünsche oder Ziele in Erfüllung gehen. Verschiedene empirische psychologische Studien haben gezeigt, dass das Ausmaß an Glück und Lebenszufriedenheit von der Verwirklichung persönlicher Lebensziele abhängig ist (vgl. Bowi 1990: 8). Je näher die Menschen ihren Wünschen oder Zielvorstellungen kommen, desto zufriedener und glücklicher scheinen sie zu sein. Der Ausgangspunkt der Wunsch- oder Zieltheorie ist somit äußerst plausibel.

Natürlich kann es bei einer Wunsch- oder Zieltheorie des guten Lebens nicht darum gehen, konkrete Rezepte oder Handlungsanweisungen zu liefern. Sie kann nicht sagen, welche inhaltlichen, beruflichen oder familiären Ziele Menschen generell wählen sollen. Die Anweisungen sind denn teilweise auch sehr vage. Der Wunschtheoretiker Martin Seel etwa rät: Man solle diejenigen Wünsche bzw. Ziele wählen, »deren Verwirklichung die reichere Erfüllung verspricht« (1995: 92). Besteht das Problem nicht gerade darin, dass wir in den meisten Fällen nicht sicher sind, welche Ziele bei ihrer Realisierung tatsächlich Erfüllung bringen? Es fehlt uns einfach schlicht die Erfahrung darüber, wie es ist, im Besitz der gewünschten Zustände oder Objekte zu sein. Der Wunsch etwa, Mutter zu werden, basiert auf der durchaus unsicheren Annahme, dass sich ein Mutterglück einstellen wird. Auch wissen wir als Schüler oder Studierende nicht >von innen<, wie es ist, einen 
bestimmten Beruf auszuüben. In entscheidenden Punkten könnte sich unser Wünschen nachträglich als blind erweisen. Man müsste dann im Nachhinein enttäuscht feststellen: Wenn ich gewusst hätte, dass das so ist, wie ich es jetzt erfahre, hätte ich dieses Ziel nicht gewählt! (vgl. Stemmer 1998: 66) Allerdings darf das kognitive Defizit, also das Nichtwissen und das damit verbundene Risiko, auch nicht überbewertet werden. Denn der Mensch wird immer schon hineingeboren in eine Gemeinschaft mit mannigfaltigen Erfahrungen und Vorstellungen bezüglich dessen, was einem Menschen Erfüllung bietet. So können wir gezielte Recherchen darüber anstellen, wie andere Menschen unter ähnlichen Lebensbedingungen ihre Mutter- oder Vaterschaft erlebt haben oder ob sie mit dem von uns anvisierten Beruf wirklich glücklich wurden. Natürlich bleibt ein Restrisiko schon aufgrund der unterschiedlichen psychischen Dispositionen und der nie ganz übereinstimmenden komplexen Lebenssituationen. Wer tiefe Wünsche oder Ziele realisieren will, ist die Strecke nicht zuvor schon einmal zur Sicherheit abgefahren. Er ist damit nicht >streckenkundig<, wie die Eisenbahner sagen.

In empirischen Studien hat man zu klären versucht, ob wir uns eher leicht erreichbare Ziele oder Ziele von hohem Schwierigkeitsgrad setzen sollen. Augenscheinlich ist es für uns nicht gut, immer die Ziele zu wählen, deren Erfolgswahrscheinlichkeit am größten ist. Denn dies wäre zweifellos bei trivialen Zielen wie Staubsaugen oder Zähneputzen der Fall: Niemand würde daran zweifeln, dass jedermann diese Ziele erreichen kann. Das Problem ist nur, dass der Wert solch trivialer Ziele sehr gering ist. Als wertvoll stufen wir normalerweise nur Ziele ein, die große Anstrengungen und hohe Qualifikationen des Handelnden erfordern. Bei solchen Zielen wäre der Schwierigkeitsgrad der Aufgabe oder des Ziels also sehr hoch. Zwischen Erfolgswahrscheinlichkeit und Wert der Ziele besteht nun offenkundig eine lineare Beziehung: Je größer die Chance der Zielerreichung ist, desto weniger Bedeutung hat der in Aussicht stehende Erfolg. Der Psychologe John Atkinson rät angesichts dieses Dilemmas zu einem gemäßigten Anspruchsniveau und mittelschweren Zielen (vgl. Rheinberg 1997: 69f.). 
Mittelschwere oder >realistische< Ziele sind attraktiv genug, um Freude zu bereiten, sind gleichzeitig aber mit vollem Einsatz der handelnden Person gerade noch zu erreichen. Wenn sich jemand durch allzu hoch gesteckte Ziele überfordert, kann es geschehen, dass die groBen Anstrengungen die Freude an den immer langsameren Fortschritten bei den nicht bewältigbaren Aufgaben überdecken. In unserer gegenwärtigen arbeitswütigen Gesellschaft besteht die Tendenz, >Raubbau an den eigenen Ressourcen $<$ zu treiben: Da das Reservoir an physischen, psychischen und intellektuellen Kräften begrenzt ist, sollte aber eine >Selbstüberbeanspruchung< oder >Selbstüberlastung< vermieden werden (vgl. Höffe 2004: 49). Wie hoch der Anreiz von schwer zu erreichenden Zielen auch immer sein mag - man muss beim Streben nach einem guten Leben die Grenzen der eigenen Ressourcen in Rechnung stellen. Jemand mit zwei linken Händen sollte sich nicht zum Ziel setzen, Goldschmiedemeister zu werden, jemand mit zwei linken Beinen nicht, Champion im Rennsport zu sein (vgl. ebd.: 44).

Für ein gutes und glückliches Leben scheint es mir grundsätzlich nicht allein auf das objektiv erreichte Niveau anzukommen. Entscheidend sind vielmehr die Möglichkeiten der Weiterentwicklung der eigenen Fähigkeiten und Kompetenzen je nach individuellem Bildungsstand und angeborenen Talenten. Es hängt also davon ab, ob sich der Einzelne bei einer bestimmten Tätigkeit kontinuierlich persönlich weiterentwickeln kann oder nicht.

\section{VERANTWORTBARE SELBSTVERWIRKLICHUNG}

Ich komme damit zum zweiten Teil und wir wenden uns dem zweiten Pol, dem der Selbsttranszendenz, zu: Viele zeitgenössische Philosophen, Psychologen und Zeitdiagnostiker warnen vor einer falschen Orientierung am >Selbst<. Die Rede von Selbstverwirklichung wecke bei oberflächlichem Verständnis »die fatale Illusion einer Weltunabhängigkeit « und verführe zu einer allzu intensiven »Beschäftigung mit sich selbst (Gerhardt 1989: 63). Je mehr es den Menschen bei ihren 
Selbstentwürfen rein um Selbstverwirklichung geht, desto mehr gerät die Selbsttranszendenz in den Hintergrund. Ein Mensch könne aber nur $\mathrm{zu}$ sich selbst finden, wenn er sich selbst transzendiert und sich den Aufgaben oder Personen in der Außenwelt hingibt. Er sei beim Projekt der Selbstverwirklichung grundsätzlich auf Selbsttranszendenz angewiesen. Doch in welchem Sinn lässt sich sagen, dass menschliche Selbstverwirklichung notwendig über den Weg der Welt führt? (Vgl. Frankl 1994: 103)

Wie bereits erwähnt, kann die menschliche Identitätsfindung und Selbstverwirklichung natürlich nur im Medium der raum-zeitlichen Wirklichkeit erfolgen, nicht etwa rein in der eigenen Phantasie. Eine echte Selbstwerdung im Unterschied zu einer Selbsttäuschung oder Lebenslüge verlangt, dass das >normative Selbstbild < in die Realität umgesetzt wird. Nur indem man seine Ziele und Ideale in der Außenwelt realisiert, kann das >normative< sukzessive in ein >empirisches Selbst< umgewandelt werden. Die Praxis der Selbstverwirklichung ist gar nicht möglich ohne die Hinwendung zur Um- und Mitwelt und das Sichten aller uns offenstehenden Handlungs- und Lebensmöglichkeiten.

Insbesondere die Mitwelt ist darüber hinaus noch in einem hermeneutischen Sinn für menschliche Selbstentwürfe grundlegend: Der Mensch kann nämlich nur in der Begegnung mit der Außenwelt zu einem Objekt werden, zu dem er sich selbst verhalten kann. In der Sozialpsychologie betont man die wichtige Rolle der gesellschaftlichen Erwartungshaltungen und Reaktionen gegenüber dem Heranwachsenden für die Herausbildung eines >Selbst< oder einer »Ich-Identität« (vgl. Mead 1993: 177-244): Nur dank der Fremdbeurteilungen durch andere Personen kann der Adoleszente die kognitive Fähigkeit entwickeln, reflexiv zu sich selbst Stellung zu beziehen. In Auseinandersetzung mit den Bildern, welche sich die anderen von ihm machen, kann er sich selbst gleichsam von außen betrachten und versuchen, einen individuellen Selbstentwurf zu entwickeln.

Die Mitmenschen können aber erfahrungsgemäß auch ein Problem für unseren Selbstentwurf werden: Sie können uns nämlich auf ein 
>empirisches Selbst< oder auf eine bestimmte Entwurfsmöglichkeit festlegen. Unter dem Blick der anderen erstarrt man gleichsam zu einem Objekt, wird von ihrem Blick fixiert und auf etwas festgenagelt. Sie kennen vielleicht Sartres Theaterstück Huis clos, Geschlossene Gesellschaft, wo die gegenseitige soziale Abhängigkeit zur Qual wird. Es heißt dort: »L'enfer, c'est les autres « - »Die Hölle, das sind die anderen«. Fatal ist es also, wenn sich Menschen nicht mehr als Subjekte begegnen, sondern sich wechselseitig verdinglichen und dadurch eine freie Selbstentfaltung vereiteln.

Halten wir fest: Die Hinwendung zur Um- und Mitwelt ist also in einem pragmatischen und hermeneutischen Sinn ganz unumgänglich. Uns interessiert heute Abend aber vor allem die ethische Dimension menschlicher Selbstentwürfe und die Frage nach der ethischen Notwendigkeit der Selbsttranszendenz. Selbsttranszendenz wird oft in einem moralischen Sinn verstanden, d.h. bezogen auf die Rücksichtnahme gegenüber den Interessen anderer Menschen. Die These lautet dann, der Mensch soll sich bei seinen Selbstentwürfen an den Anforderungen oder Problemen in seiner Um- und Mitwelt orientieren, statt nur an seine eigenen Bedürfnisse und Wünsche zu denken. Warum sollen wir uns aber um das Allgemeinwohl kümmern statt nur um das für uns selbst Gute? Verlieren wir nicht uns selbst, wenn wir uns an den Bedürfnissen und Interessen anderer Menschen oder an Weltproblemen orientieren? Oder gewinnen wir ganz im Gegenteil nur so uns selbst und sind nur dann wahrhaft Mensch?

Manche von Ihnen denken vielleicht: Diese Fragen sind doch ganz einfach zu beantworten, wenn man sich die kategorischen Forderungen der Sozialethik oder Moralphilosophie vergegenwärtigt. Sie erinnern sich an meine einführenden Erläuterungen zu den beiden Perspektiven in der Ethik: Auf die ethische Grundfrage, wie wir handeln sollen, antwortet die Moralphilosophie klar und deutlich: Wir sollen den egoistischen Interessenstandpunkt aufgeben und den unparteiischen objektiven Standpunkt der Moral einnehmen. Von diesem Standpunkt aus erscheint das Wohlergehen von Um- und Mitwelt als genauso wichtig wie das eigene. Doch viele von Ihnen werden sich mit dieser 
Antwort nicht zufrieden geben und weiterfragen: Ja, warum sollen wir Menschen überhaupt moralisch sein? Wollen wir nicht primär glücklich sein, was nur über eine gelingende Selbstverwirklichung möglich scheint? Ist die Rücksichtnahme auf fremde Interessen und die Beschränkung unseres Handelns durch moralische Verbote nicht hinderlich beim Streben nach Selbstverwirklichung?

Der Philosoph Michael Theunissen hat eine Studie mit dem Titel Selbstverwirklichung und Allgemeinheit verfasst. Ihm zufolge orientiert sich eine wahre, vernünftige Selbstverwirklichung an den allgemeinen Problemen dieser Welt: an der weltweiten Ausbeutung der Natur, dem Hunger in der Welt und der Bedrohung des Weltfriedens. Ich zitiere: »Die Selbstverwirklichung, die von uns gefordert ist, konkretisiert sich heute also in der Bekümmerung um die weltweite Ausbeutung der Natur, in der Betroffenheit vom Hunger in der Welt, in der Sorge um den Weltfrieden.« (Theunissen 1981: 46)

Ähnlich lautet die These Friedrich Kambartels in seinem Referat zum Thema Universalität als Lebensform. Wer sich selbst verwirklichen und ein gutes Leben führen wolle, müsse sich an überindividuellen Zielen orientieren. Auch ihn möchte ich kurz zitieren: »Um in einem wesentlichen, praktischen Sinne zu uns selbst zu gelangen, müssen wir unsere (individuelle) Subjektivität überwinden. [...] Das vernünftige Leben ist das gemeinsame gute Leben.« (Kambartel 1978: 20) Kambartel beschränkt den Horizont der gemeinsamen Ziele zunächst auf diejenigen Menschen, die in einem gemeinsamen Handlungszusammenhang stehen. Aufgrund der Ausweitung dieses Handlungszusammenhangs im Zeichen der Globalisierung sei aber das gute Leben inzwischen ein Menschheitsprojekt geworden (ebd.: 19).

Bei Otfried Höffe finden wir die gleiche Argumentationsstrategie, aber etwas konkreter ausgeführt (vgl. Höffe 2004: 52f.): Der Mensch ist ein soziales Wesen und ist bei seinem Streben nach Glück und Selbstverwirklichung vielfältig auf ein intaktes soziales Umfeld angewiesen. Die meisten Ziele können wir nur dank des Wohlwollens und der Unterstützung von Freunden, Partnern oder Kollegen verwirklichen. Deren eigenes Wohlergehen ist uns nicht gleichgültig, sondern 
trägt zum eigenen Wohlergehen bei. Denn wenn es ihnen schlecht geht, geht es auch uns schlecht. Tragfähige soziale Beziehungen erfordern gegenseitige Hilfsbereitschaft, Wohlwollen und Solidarität. Man hilft den anderen dann uneigennützig, also ohne weitere Nützlichkeitserwägungen. Man tut das Gute um des Guten willen. Soweit ist der Gedankengang sicherlich unproblematisch und leicht nachvollziehbar.

Ähnlich wie Kambartel bedient sich Höffe darüber hinaus eines sogenannten Ausdehnungs-Arguments, um moralisches Handeln vom Nahbereich auf den Fernbereich zu erweitern. Natürlich sind wir nicht nur auf unsere Freunde und unsere Familie im Nahbereich angewiesen, sondern auf geordnete und friedliche soziale Verhältnisse in der Gesellschaft, in der wir leben. Neuere sozialwissenschaftliche Studien haben klar nachgewiesen, dass in einer Gesellschaft ohne gemeinsame moralische Werte und Normen der Zusammenhalt fehlt. Eine Gesellschaft von egoistischen Selbstverwirklichern würde über kurz oder lang in einen anarchistischen Zustand wie bei Thomas Hobbes' Krieg aller gegen alle münden. Um uns selbst zu verwirklichen und unser Glück zu machen, brauchen wir humanitäre Arbeitsverhältnisse, eine florierende Wirtschaft mit einem vielfältigen Angebot an Arbeitsplätzen, Dienstleistungsbetriebe, Verkehrsnetze, Bildungsinstitutionen und vieles weitere mehr.

Auch Höffe betont, dass die Handlungszusammenhänge in der heutigen Gesellschaft schnell die ganze Menschheit umfassen können. Aufgrund der weitreichenden wirtschaftlichen, gesellschaftlichen und politischen Verflechtungen bedürfe es der Solidarität auch mit den Bewohnern entfernterer Regionen und mit den zukünftigen Generationen. Er macht aufmerksam auf unsere wirtschaftliche Abhängigkeit von ausländischen Märkten hinsichtlich der Rohstoff- und Energiequellen. Die weltweite Verflochtenheit im politischen Bereich illustriert Höffe anhand von politischen Unruhen in weit entfernten Ländern, die über internationale Netzwerke auf andere Regionen übergreifen können. Schließlich führt er die sich verschärfende Umweltproblematik ins Feld, welche eine Verantwortlichkeit gegenüber zukünftigen Generationen erforderlich mache: Zunehmende Verschmutzung 
der Gewässer und der Luft, die Ausrottung von Tier- und Pflanzenarten und die Klimaerwärmung drohen zukünftiges Leben auf der Erde unmöglich zu machen. Ist damit aber wirklich gezeigt, dass unser persönliches Glück von der Solidarität mit unseren Fernsten und vom Wohl zukünftiger Generationen abhängt?

Aus kritischer Distanz kann die Ableitung der moralischen Forderung nach Selbsttranszendenz aus einer allmählichen Ausweitung der Handlungszusammenhänge nicht wirklich überzeugen. Es fehlt ihnen letztlich die normative Kraft, wie Höffe selbst gesteht. Denn die gravierenden >Weltprobleme< wie Hungersnöte oder Umweltkrisen sind gerade von der Art, dass sie den Einzelnen und sein persönliches Glücksstreben kaum beeinträchtigen. So handelt es sich bei den meisten Umweltschäden um >Kumulationsprobleme $<$, die sich als Folge nicht-koordinierten Handelns vieler Akteure ergeben. Die individuellen Beiträge der Einzelnen, wie etwa die eigenen Autoabgase, fügen den Verursachern selbst keinen spürbaren Schaden zu. Die reichen Industrienationen im gemäßigten Norden sind von den langfristigen globalen Folgen der Klimaerwärmung nicht direkt betroffen. So bleiben die meisten Menschen unbeschwert und erkennen keine moralische Pflicht. Es scheint sich hier vielmehr eine parasitäre Haltung anzubieten: Der Einzelne kann daraus Vorteile ziehen, dass sich die meisten anderen an die allgemeinen Umweltgebote halten. Desgleichen beeinträchtigt ein Krieg in Nigeria oder im Nahen Osten mein individuelles gutes Leben schwerlich. Es lässt sich argumentativ nicht zeigen, dass eine Selbstverwirklichung ohne individuelles Engagement für Weltprobleme notwendigerweise zum Scheitern verurteilt ist.

Andererseits gibt es sehr wohl individualethische Gründe dafür, die persönlichen Selbstentwürfe an dem für alle Guten oder moralisch Richtigen zu orientieren. Dies sind allen voran die drohenden moralischen Sanktionen wie Tadel, Verachtung oder Ausgrenzung durch die Gemeinschaft. Wer offenkundig und permanent auf Kosten anderer Menschen nach Glück strebt und sich rücksichtslos entfaltet, wird von der moralischen oder rechtlichen Gemeinschaft über kurz oder lang in die Schranken gewiesen. Auch wo das unmoralische Handeln unent- 
deckt bleibt, lebt man in ständiger Furcht, dass es irgendwann doch noch auskommt. Darüber hinaus können wir nur durch soziale Beziehungen wechselseitiger Anerkennung und des Respekts eine positive Selbstbeziehung aufrecht halten. Nach Axel Honneth lassen sich drei Ebenen der Anerkennungsverhältnisse unterscheiden: Das für unsere individuelle Selbstverwirklichung sehr wichtige Selbstvertrauen durch Anerkennung in der Familie, Selbstachtung durch die Rechtsgemeinschaft und Selbstschätzung durch die Solidargemeinschaft (vgl. Honneth 1998: 45f.).

Es ist aber nicht so wie von antiken Philosophen gern behauptet, dass ein gerechtes Leben in Hingabe an dringliche Aufgaben in der Welt automatisch glücklich macht. Wohl gibt es viele Menschen, die sich das Engagement für Hilfsbedürftige oder für die Umwelt zum zentralen Lebensziel machen und darin Erfüllung und Glück finden. Auch kann das Selbstverwirklichungsstreben indirekt der Gesellschaft nützen, so z.B. bei vielen Künstlern und Denkern, die die Welt mit ihren Werken beglücken. Das für mich Gute muss also keineswegs immer im Widerspruch stehen zum sozialethisch Guten. Es kann aber durchaus auch sein, dass die Forderungen der Um- und Mitwelt eine Einschränkung der Selbstverwirklichung und der Verfolgung individueller Ziele erfordern.

Ethisch gesehen ist grundsätzlich nur eine Selbstverwirklichung angemessen, die auf die Um- und Mitwelt Rücksicht nimmt. Es ist typisch für den Menschen, dass er sich in die Lage der anderen versetzen und gleichsam die Außenperspektive auf sich selbst einnehmen kann. Deswegen ist der Mensch das einzige Lebewesen, das Verantwortung für sein Handeln gegenüber den Betroffenen übernehmen kann. Gerade weil die Menschen frei sind, ihr Leben zu führen und sich selbst zu entwerfen, erwarten wir voneinander eine Rechtfertigung und Begründung unseres Tuns. Zum Menschsein gehört also wesentlich, den moralischen Standpunkt einzunehmen und Verantwortung gegenüber den vom eigenen Handeln Betroffenen zu übernehmen. Dem Menschen ist nur eine verantwortbare Selbstverwirklichung angemessen, bei der die 
Verwirklichung der eigenen Interessen mit Blick auf fremde Interessen begrenzt wird.

Der Anlage nach ist die Fähigkeit zur Selbsttranszendenz und zur Fremdzentrierung zwar genauso in der menschlichen Natur verankert wie der Egozentrismus. Anders als das egozentrische Streben nach Bedürfnis- und Wunschbefriedigung ist die menschliche Fähigkeit zur Selbsttranszendenz und zum Altruismus aber nicht von Geburt an aktuell. Sie fehlt bekanntlich beim Neugeborenen. Sie entwickelt sich erst im Heranwachsenden und ist auf sorgfältige moralische Erziehung und Sozialisation angewiesen. Je mehr Heranwachsende zur Wahrnehmung der Weltprobleme erzogen oder für sie sensibilisiert werden, desto größer ist die Chance eines selbstlosen Engagements für Weltprobleme. Wem die Selbsttranszendierung zum festen Charakter geworden ist, der tut das moralisch Richtige ganz unabhängig vom Eigennutz. Eine verantwortungsvolle und wahrhaft menschliche Selbstverwirklichung ist also ohne Selbsttranszendenz nicht möglich. Und erst durch eine moralische Erziehung und Bildung wird der Mensch als zur Reflexion begabtes Naturwesen zum Menschen.

\section{LITERATUR}

Aristoteles (1991): Nikomachische Ethik, München.

Bowi, Ulrike (1990): Der Einfluss von Motiven auf Zielsetzung und Zielrealisierung, Dissertation, Heidelberg.

Frankl, Viktor (1994): Logotherapie und Existenzanalyse, Berlin/München.

Gehlen, Arnold (1986): Der Mensch. Seine Natur und seine Stellung in der Welt, Wiesbaden.

Gerhardt, Gerd (1989): Kritik des Moralverständnisses, Bonn.

Gewirth, Alan (1998): Self-fulfillment, Princeton.

Höffe, Otfried (2004): Das Glück, Kurseinheit 1 des Studienbriefs »Persönliches Glück und politische Gerechtigkeit«, Hagen. 
Honneth, Axel (1998): Kampf um Anerkennung. Zur Grammatik sozialer Konflikte, Frankfurt a.M.

Horn, Christoph (2014): »Philosophische Glückstheorien. Systematische und historische Überlegungen«, in: Monika Müller/Stephan Schaede et.al. (Hg.), Was ist ein gutes Leben? Mehr als eine flüchtige Frage nach dem schnellen Glück, Loccum, S. 73-92.

Kambartel, Friedrich (1978): »Universalität als Lebensform«, in: Willi Ölmüller (Hg.), Normenbegründung und Normendurchsetzung, Bd. 2, Paderborn, S. 11-21.

Kleiter, Ekkehard (1999): Egozentrismus, Selbstverwirklichung und Moral, Bd. 1, Weinheim.

Krämer, Hans (1978): »Selbstverwirklichung«, in: Günther Bien (Hg.), Die Frage nach dem Glück, Stuttgart-Bad Cannstatt, S. 21-44.

Mead, Herbert (1993): Identität und Gesellschaft, Frankfurt a.M.

Nietzsche, Friedrich (1999): »Jenseits von Gut und Böse. Vorspiel zu einer Philosophie der Zukunft«, in: ders.: Sämtliche Werke. Kritische Studienausgabe (KSA), Bd. 5, München, S. 9-244.

Rheinberg, Falko (1997): Motivation, Stuttgart/Berlin/Köln.

Sartre, Jean-Paul (1989): Ist der Existentialismus ein Humanismus? Drei Essays, Frankfurt a.M.

Sartre, Jean-Paul (1991): Das Sein und das Nichts. Versuch einer phänomenologischen Ontologie, Reinbek bei Hamburg.

Scheler, Max (1991): Die Stellung des Menschen im Kosmos, Bonn.

Seel, Martin (1995): Versuch über die Form des Glücks, Frankfurt a.M.

Stemmer, Peter (1998): »Was es heisst, ein gutes Leben zu leben«, in: Holmer Steinfath (Hg.), Was ist ein gutes Leben?, Frankfurt a.M., S. 47-72.

Theunissen, Michael (1981): Selbstverwirklichung und Allgemeinheit. Zur Kritik des gegenwärtigen Bewusstseins, Berlin/New York. 